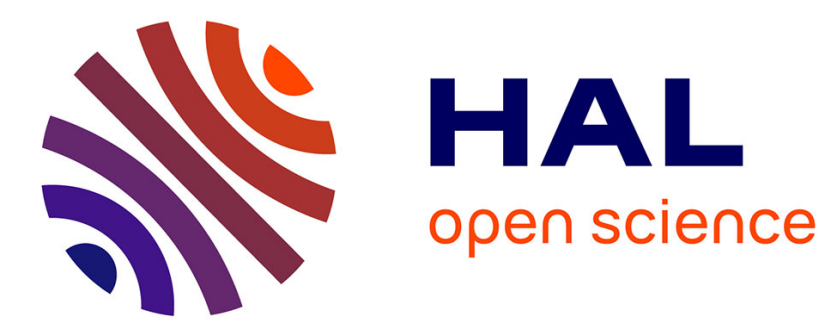

\title{
Magnetic and magnetocaloric properties of the high-temperature modification of TbTiGe
}

Sophie Tencé, Etienne Gaudin, Olivier Isnard, Bernard Chevalier

\section{To cite this version:}

Sophie Tencé, Etienne Gaudin, Olivier Isnard, Bernard Chevalier. Magnetic and magnetocaloric properties of the high-temperature modification of TbTiGe. Journal of Physics: Condensed Matter, 2012, 24 (29), pp.296002. 10.1088/0953-8984/24/29/296002 . hal-00712605

\section{HAL Id: hal-00712605 \\ https://hal.science/hal-00712605}

Submitted on 28 Jun 2012

HAL is a multi-disciplinary open access archive for the deposit and dissemination of scientific research documents, whether they are published or not. The documents may come from teaching and research institutions in France or abroad, or from public or private research centers.
L'archive ouverte pluridisciplinaire HAL, est destinée au dépôt et à la diffusion de documents scientifiques de niveau recherche, publiés ou non, émanant des établissements d'enseignement et de recherche français ou étrangers, des laboratoires publics ou privés. 


\section{Magnetic and magnetocaloric properties of the high temperature modification of TbTiGe}

S. Tencé ${ }^{1}$, E. Gaudin ${ }^{2}$, O. Isnard ${ }^{1}$, B. Chevalier ${ }^{2,3}$

${ }^{1}$ Institut Néel, CNRS/Université Joseph Fourier, B.P. 166, 38042 Grenoble France

${ }^{2}$ CNRS, Université de Bordeaux, ICMCB, 87 avenue du Docteur Albert Schweitzer, 33608

Pessac Cedex, France

E-mail :chevalie@icmcb-bordeaux.cnrs.fr

${ }^{3}$ Author to whom any correspondence should be addressed 


\begin{abstract}
The high temperature form (HT) of the ternary germanide TbTiGe was prepared by melting. The investigation of HT-TbTiGe by X-ray and neutron powder diffractions shows that the compound crystallizes in the tetragonal CeScSi-type structure (space group I4/mmm; $a=$ 404.84(5) and $c=1530.10(9) \mathrm{pm}$ as unit cell parameters). Magnetization and specific heat measurements as well as neutron powder diffraction performed on HT-TbTiGe reveal a ferromagnet having $\mathrm{T}_{\mathrm{C}}=300(1) \mathrm{K}$ as Curie temperature; the $\mathrm{Tb}$-moments are aligned along the $c$-axis. This magnetic ordering is associated to a modest magnetocaloric effect around room temperature. The isothermal magnetic entropy change $\Delta S_{m}$ was determined from the magnetization data; $\Delta S_{m}$ reaches respectively a maximum value of -4.3 and $-2.0 \mathrm{JK}^{-1} \mathrm{~kg}^{-1}$ for a magnetic field change of 5 and $2 \mathrm{~T}$.
\end{abstract}

Keywords: Terbium, Ternary germanide; Structural properties; Ferromagnetism; Magnetic structure; Magnetocaloric properties

PACS numbers: 61.05.cp, 61.05.F-, 75.25.-j, 75.30.Sg, 75.50.Cc 


\section{Introduction}

The investigation of the isothermal section at $1070 \mathrm{~K}$ of the Tb-Ti-Ge system reveals that the structural properties of the ternary germanide TbTiGe are depending on the temperature [1]. In this last reference [1], it was reported that the high temperature (HT) modification (tetragonal CeFeSi-type; space group $P 4 / \mathrm{nmm}$ ) was obtained by quenching from the liquid during electric arc furnace melting whereas the low temperature (LT) modification (tetragonal CeScSi-type; space group I4/ $\mathrm{mmm}$ ) was detected in the sample after annealing at $1070 \mathrm{~K}$. But after this last thermal treatment, the two HT and LT phases coexist. These results were confirmed by Welter et al. [2] who have investigated the crystallographic properties of the HT modification (CeFeSi-type) using X-ray diffraction on a single crystal extracted from the ascast ingot of TbTiGe. To date, the crystal structure of the TbTiGe ternary compound adopting the CeScSi-type was determined by X-ray powder diffraction performed on a sample containing a mixture of the two HT and LT modifications [1].

Recently, we have shown that the ternary germanide CeTiGe exhibits a structural phase transition as a function of the temperature, the tetragonal HT and LT modifications adopting respectively the $\mathrm{CeScSi}$ - and CeFeSi-type [3]. This behavior is opposite to that previously reported for TbTiGe. It is interesting to note that this structural change influences the magnetic properties of CeTiGe. A decrease of the hybridization coefficient $J_{c f}$ between $4 f(\mathrm{Ce})$ and conduction electrons is evidenced in the sequence LT-CeTiGe (CeFeSi-type) $\rightarrow$ HTCeTiGe (CeScSi-type); the HT modification is classified as a non-magnetic strongly correlated electron system [3] as shown by an enhanced Sommerfield coefficient $\gamma=0.308 \mathrm{~J}$ $\mathrm{mol}^{-1} \mathrm{~K}^{-2}$.

More recently, we have shown that the ternary germanide GdTiGe can be obtained as single phase crystallizing in the CeScSi-type by melting of the elements followed by a quenching [4]. This study agrees with that performed on CeTiGe revealing that the HT modification of the $R E \mathrm{TiGe}(R E=$ rare earth) compounds adopts the tetragonal CeScSi-type.

In this view, it is interesting to obtain as pure phase the crystallographic form of TbTiGe adopting the CeScSi-type and to determine its magnetic properties. We remind that an antiferromagnetic ordering around 286-288 $\mathrm{K}$ was reported for TbTiGe crystallizing in the tetragonal CeFeSi-type [5-7]. In this paper, we report on the synthesis of the two forms HT and LT of TbTiGe. The first one (CeScSi-type) was much less investigated in the past, we consequently present a more detailed study of this HT form, using X-ray and neutron powder diffractions, specific heat and magnetization measurements. Also, the magnetic structure and 
the magnetocaloric properties of this HT modification of TbTiGe are determined and discussed in relation to those reported previously for the LT modification showing an antiferromagnetic behaviour.

\section{Experimental}

Sample with the following composition $32 \% \mathrm{~Tb}: 35 \% \mathrm{Ti}: 33 \% \mathrm{Ge}$ as atomic ratio percentage was prepared by melting precisely weighted amounts of high purity elements $\mathrm{Tb}$, $\mathrm{Ti}$ and Ge $(99.9 \%)$ in a levitation induction furnace. This starting composition was deduced from the phase equilibrium in the Tb-Ti-Ge system [1] in order to inhibit the presence of $\mathrm{Tb}_{5} \mathrm{Ge}_{3}$ or $\mathrm{Tb}_{5} \mathrm{Ge}_{4}$ (ordered magnetic compounds) in the final sample. Melting was performed several times to ensure a good homogeneity, in a water-cooled copper crucible, under a purified argon atmosphere. The weight losses during the overall melting process were less than 0.1 wt. $\%$ and finally the as-cast sample (HT-TbTiGe) was quenched by switching off the power supply. Then, the annealed sample (LT-TbTiGe) was obtained after thermal treatment of a part of the as-cast sample enclosed in evacuated quartz tube at $1073 \mathrm{~K}$ for seven weeks. No reaction between the sample and the quartz tube was observed.

Both the composition and homogeneity of the as-cast and annealed samples were checked by microprobe analysis using a Cameca SX-100 instrument. The analysis was performed on the basis of intensity measurements of $\mathrm{Tb}-\mathrm{L} \alpha_{1}, \mathrm{Ti}-\mathrm{K} \alpha_{1}$ and $\mathrm{Ge}-\mathrm{K} \alpha_{1} \mathrm{X}$-rays emission lines, which were compared with those obtained for the high purity elements $\mathrm{Tb}$, Ti and Ge used as reference materials.

X-ray powder diffraction was performed with the use of a Philips 1050-diffractometer (Cu$\mathrm{K} \alpha$ radiation) for the characterization of the structural type and for the phase identification of the as-cast and annealed samples. To determine the crystal structure of HT-TbTiGe, X-ray patterns were recorded at room temperature with a high-resolution Bruker D8 powder diffractometer in transmission geometry $\left(10^{\circ}<2 \theta<100^{\circ}\right.$, step size of $\left.0.02^{\circ}\right)$. It operates with a focusing $\mathrm{Ge}(111)$ monochromator selecting the $\mathrm{Cu}-\mathrm{K} \alpha_{1}$ radiation and eliminates the fluorescence by using a Moxtek-type solid state detector.

Neutron diffraction experiments were carried out at the high flux reactor of the Institut Laue Langevin. (Grenoble, France) using the high flux powder diffractometer D1B operated by the French CNRS. The neutron diffraction patterns as function of temperature were obtained in the range of 1.5 to $310 \mathrm{~K}$. The neutron wavelength of $2.52 \AA$ was selected. All 
powder neutron diffraction data were analyzed using the Rietveld line profile technique implemented with the program FULLPROF [8]. A pseudo-Voigt type peak shape function was used in the refinements and, in the case of the D1B data, the peak shape was very close to pure Gaussian. The coherent neutron scattering lengths were $b_{\mathrm{Tb}}=0.73810^{-12} \mathrm{~cm}, b_{\mathrm{Ti}}=-$ $0.343810^{-12} \mathrm{~cm}$, and $b_{\mathrm{Ge}}=0.818510^{-12} \mathrm{~cm}$ for $\mathrm{Tb}$, Ti and Ge, respectively [9]. A full description of the Rietveld method used in this article as well as the definition of the agreement factors can be found elsewhere [10].

Magnetization measurements were performed using a superconducting quantum interference device (SQUID) magnetometer (Quantum Design MPMS-XL) in the temperature range 2-350 $\mathrm{K}$. The field dependence at $10 \mathrm{~K}$ of the magnetization for HT-TbTiGe was performed at ambient pressure using the extraction method in applied fields from zero to 10 $\mathrm{T}$ [11]. The same type cryostat was used with another insert enabling any temperature between 300 and $850 \mathrm{~K}$ to be regulated. This set up has been used to investigate the magnetic properties of HT-TbTiGe above room temperature [12] and in this case the maximum field used was 1T.

Heat capacity was determined with a standard relaxation method with a Quantum Design PPMS device. Sample of approximately $40 \mathrm{mg}$ was glued to the sample holder using Apiezon $\mathrm{N}$-grease. The heat capacity of the sample holder and grease was measured just before the sample was studied.

\section{Results and discussion}

\subsection{Structural properties from X-ray diffraction investigation}

Microprobe analysis performed on the as-cast and annealed samples indicates their high chemical purity. These samples contain a major phase TbTiGe ( $>99 \%$ in volume) with some amounts of the binary compound $\mathrm{Ti}_{5} \mathrm{Ge}_{3}$. The presence of this last binary germanide agrees with the choice of the nominal composition $(32 \% \mathrm{~Tb}: 35 \% \mathrm{Ti}: 33 \% \mathrm{Ge}$ as atomic ratio percentage) used for the synthesis of the samples.

The comparison of the X-ray powder patterns obtained on the as-cast or annealed TbTiGe sample evidences strong differences (Figure 1). For instance, the strong peak observed near $2 \theta=28^{\circ}$ on the pattern of the as-cast sample, disappears after annealing. This analysis reveals that TbTiGe undergoes a structural transition as a function of temperature. After melting (HT 
modification) TbTiGe crystallizes in the tetragonal CeScSi-type structure (space group $I 4 / \mathrm{mmm}$ ) whereas after annealing (LT modification) the ternary germanide adopts the tetragonal CeFeSi-type structure (space group $P 4 / \mathrm{nmm}$ ). This structural transition evidenced for TbTiGe is similar to that reported by us during the synthesis of CeTiGe [3]. On the contrary, the results obtained in our study are in disagreement with those reported previously $[1,2]$.

The Rietveld analysis of the X-ray powder pattern of HT-TbTiGe recorded at room temperature confirms that it crystallizes with the CeScSi-type structure (space group $14 / \mathrm{mmm}$ ) (Figure 2). Some extra weak peaks correspond to the presence of a small amount ( $\approx 4 \mathrm{wt} \%)$ of the impurity phase $\mathrm{Ti}_{5} \mathrm{Ge}_{3}$, in agreement with the microprobe analysis. (At this stage it is important to mention that $\mathrm{Ti}_{5} \mathrm{Ge}_{3}$ shows a weak Pauli paramagnetism [13] therefore its presence will not influence the magnetic properties of the HT-TbTiGe sample). The unit cell parameters are refined to $a=404.84(5)$ and $c=1530.10(9) \mathrm{pm}, \mathrm{i}$. e. they are slightly smaller than those of GdTiGe ( $a=406.45(5)$ and $c=1544.8(2) \mathrm{pm})$ [4] which is consistent with the lanthanide contraction. The remaining discrepancies between calculated and measured intensities come mostly from the existence of preferred orientation, visible for instance on the (001) lines which are too small in comparison to the (110) or (200) lines without any correction. Therefore, the preferred orientation has been taken into account and partly corrected to improve the refinement. The final Rietveld refinement of the atomic positions with the isotropic atomic displacement parameters leads to the reliability factor $\mathrm{R}_{\mathrm{Bragg}}=6.7 \%$ and the profile factors $R_{p}=13.2 \%$ and $R_{w p}=12.0 \%$ (Figure 2). These structural parameters are gathered in Table 1.

At this stage it is important to mention that $\mathrm{Ti}_{5} \mathrm{Ge}_{3}$ shows a weak Pauli paramagnetism therefore its presence will not influence the magnetic properties of two samples

The positional parameters determined here for the HT-TbTiGe ternary germanide agree with those reported previously for this compound during the investigation by X-ray powder diffraction of a mixture containing HT- and LT-TbTiGe [1]. The comparison between the interatomic distances d existing respectively in the HT- [this work] and the LT- [2] forms of TbTiGe shows: (i) a small increase of the $\mathrm{d}_{\mathrm{Tb}-\mathrm{Tb}}$ distances from 370.4 to $371.4 \mathrm{pm}$ in the sequence LT-TbTiGe $\rightarrow$ HT-TbTiGe and (ii) a decrease of the $\mathrm{d}_{\mathrm{Tb}-\mathrm{Ge}}(298.0 \rightarrow 294.4 \mathrm{pm})$ and $\mathrm{d}_{\mathrm{Tb}-\mathrm{Ti}}(335.0 \rightarrow 332.9 \mathrm{pm})$ distances in the same sequence. These changes could lead to different magnetic behavior for the two crystallographic forms of TbTiGe. 


\subsection{Magnetic properties}

The temperature dependence of the magnetization $M$ of HT-TbTiGe divided by low applied magnetic field $H=0.05 \mathrm{~T}$ is strongly dependent upon its cooling history as observed by the occurrence of a strong irreversibility between the zero field cooled (ZFC) and field cooled (FC) curves (Figure 3). The features characteristic of a ferromagnetic ordering are clearly seen in the data near $\mathrm{T}_{\mathrm{C}}=300(1) \mathrm{K}$ (temperature defined by a minimum in the derivative curve $\left.\left(\mathrm{d} M_{\mathrm{ZFC}} / \mathrm{H}\right) / \mathrm{dT}\right)$. A broad maximum is observed around $275(5) \mathrm{K}$ just below $\mathrm{T}_{\mathrm{C}}$ in the ZFC curve, which is followed by a drastic fall in the $M$ value, whereas the FC curve continuously rises with decreasing temperature. In the ferromagnetic systems, a bifurcation of ZFC-FC curves appears if the coercive field becomes larger than the applied magnetic field (see below). Also, such a bifurcation is observed in anisotropic systems based for instance on rare earth having an orbital moment $(\mathrm{Nd}, \mathrm{Tb}, \ldots)$. This study suggests that the HT-TbTiGe phase exhibits a ferromagnetic behaviour as reported previously for the GdTiGe compound crystallizing in the CeScSi-type [4, 14].

On the contrary, the $M / H$ versus $\mathrm{T}$ curve corresponding to the LT-TbTiGe phase (inset of Figure 3) shows a maximum near $\mathrm{T}_{\mathrm{N}}=280(1) \mathrm{K}$ characteristic of the occurrence of an antiferromagnetic ordering. The Néel temperature $T_{N}$ determined by us is close to that reported previously in ref. [5, 7] $(286-288 \mathrm{~K})$ but smaller than that observed in ref. [6] $(312$ $\mathrm{K})$. But with this investigation, it is clear that the structural transition CeScSi-type (HT$\mathrm{TbTiGe}) \rightarrow$ CeFeSi-type (LT-TbTiGe) induces a magnetic transition from ferromagnetism to antiferromagnetism.

As shown in Figure 4 the temperature dependence of the reciprocal magnetic susceptibility $\chi_{\mathrm{m}}{ }^{-1}=\mathrm{f}(\mathrm{T})$ of HT-TbTiGe compound follows a Curie-Weiss law above $310 \mathrm{~K}$ with a value of the paramagnetic Curie temperature $\theta_{\mathrm{p}}$ equal to $285(2) \mathrm{K}$. A paramagnetic behaviour is then observed in this temperature range, and the positive value of $\theta_{p}$ confirms the ferromagnetic ordering of this ternary germanide. The experimental value of the effective magnetic moment $\mu_{\text {eff. }}=10.41(5) \mu_{\mathrm{B}} / \mathrm{Tb}$ is slightly larger than the theoretical free ion value of $9.70 \mu_{\mathrm{B}} / \mathrm{Tb}$ for

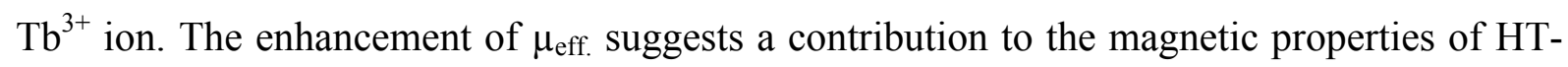
TbTiGe compound from the polarization of the conduction electrons as observed for instance in the ternary stannide $\mathrm{Tb}_{6} \mathrm{Co}_{2.35} \mathrm{Sn}_{0.65}[15]$. 
At $10 \mathrm{~K}$, the hysteresis curve $M$ versus $\mu_{0} H$ for HT-TbTiGe (Figure 5) agrees with the behaviour of a polycrystalline ferromagnet. The loop exhibits an important coercitive field of $1 \mathrm{~T}$ and the magnetization $M$ is not completely saturated at $10 \mathrm{~T}$ revealing most probably a significant magnetocrystalline anisotropy. In these last conditions, $M$ is close to 7.79(5) $\mu_{\mathrm{B}} /$ mol, a value smaller than that expected for a $\mathrm{Tb}^{3+}$ moment $\left(\mathrm{g}_{\mathrm{J}} \mathrm{J}=9.0 \mu_{\mathrm{B}} / \mathrm{Tb}\right)$.

In order to confirm the conclusions of the magnetization measurements, specific heat $C_{p}$ measurements were performed on the HT-TbTiGe sample. The temperature dependence of $\mathrm{C}_{\mathrm{p}}$ in zero applied magnetic field is shown on Figure 6 . The $\mathrm{C}_{\mathrm{p}}$ versus $\mathrm{T}$ curve exhibits only a $\lambda$ type peak; its maximum is around $289(2) \mathrm{K}$ and with decreasing temperature $\mathrm{C}_{\mathrm{p}}$ starts to increase near 299(2) K. These results are in excellent agreement with the magnetization data and confirm that HT-TbTiGe has only one magnetic transition below $320 \mathrm{~K}$. It is interesting to note that the $\lambda$-peak observed here for the ferromagnetic HT-TbTiGe compound is much higher than that reported for the antiferromagnetic LT-TbTiGe [16]. In addition to the different nature of the magnetic structure, this can also indicate that the quality of the two samples is different.

\subsection{Nuclear and magnetic structures from neutron diffraction investigation}

A neutron diffraction pattern was recorded at $310 \mathrm{~K}$, namely above the Curie temperature $\mathrm{T}_{\mathrm{C}}$, in order to only have the nuclear contribution and to determine precisely the crystallographic structure of HT-TbTiGe modification. At this temperature, all Bragg peaks can be indexed with the space group $I 4 / \mathrm{mmm}$. It is worth noticing that small peaks resulting from the $\lambda / 2$ harmonics ( $0.8 \%$ of the $\lambda$ contribution) are present and taken into account in the refinements. The model using the CeScSi-type structure and as starting atomic coordinates the values of the X-ray diffraction converge immediately to a similar solution. Then, the refined parameters are $a=404.87(1)$ and $c=1529.62(7) \mathrm{pm}, \mathrm{z}_{\mathrm{Tb}}=0.3255(3)$ and $\mathrm{z}_{\mathrm{Ge}}=0.1249(2)$, values in fair agreement with the X-ray data at room temperature (Table 1). The accuracy of the atom position is somewhat limited by the preferred orientation, in particular for X-ray diffraction measurements a technique more sensitive to preferred orientation. At this point it is worth to remark also that a large contrast of neutron scattering length exists between the elements and even a negative sign for the $\mathrm{Ti}$ nucleus opposite to that of $\mathrm{Tb}$ and $\mathrm{Ge}$. Consequently the structural parameters deduced from neutron diffraction can be expected to be more precise 
than that derived above from $\mathrm{X}$ ray diffraction. In addition, no significant deviation from the stoechiometric composition is observed by neutron diffraction.

Below $\mathrm{T}_{\mathrm{C}}$, magnetic contributions appear on the nuclear peaks indicating the onset of a ferromagnetic state in agreement with the magnetization measurements. Thus, all the observed magnetic reflections can be indexed with the propagation vector $\mathrm{k}=(000)$ down to 2 K. According to the magnetic symmetry analysis, used to determine the irreducible representations (IR) with their basis vectors, only four IR are allowed for the $\mathrm{Tb}$ site $4 e$ for $\mathrm{k}$ $=(000)$ and the space group $I 4 / \mathrm{mmm}$. Only two IR correspond to a ferromagnetic structure: one with the Tb-moments along the $c$-axis and one with the Tb-moments within the basal ( $a$, b)-plane. Since there is no magnetic contribution on the reflections (001), the solution is likely the former one. Indeed, the model with magnetic Tb-moments parallel to the $c$-axis leads to the best refinement of the data with $\mathrm{R}_{\mathrm{B}-\mathrm{nucl}}=9.8 \%$ and $\mathrm{R}_{\mathrm{B}-\mathrm{mag}}=7.6 \%$. Therefore, the magnetic structure consists of ferromagnetic Tb-planes ferromagnetically coupled with next adjacent $\mathrm{Tb}$-planes along the $c$-axis (Figure 7). Figure 8 shows the final refinement for which the Tb-moment value amounts to $8.90(4) \mu_{\mathrm{B}}$ at $2 \mathrm{~K}$. This value corresponds to the trivalent state of the $\mathrm{Tb}^{3+}$ ions $\left(\mu_{\mathrm{Tb}}{ }^{+3}=9.00 \mu_{\mathrm{B}}\right)$ and is slightly higher than the maximum value of $M=$ $7.79(5) \mu_{\mathrm{B}}$ deduced from the magnetization curve recorded at $10 \mathrm{~K}$ (Figure 5). This difference is most probably due to the fact that the magnetization is not completely saturated at $10 \mathrm{~T}$.

The different patterns recorded between 2 and $310 \mathrm{~K}$ allow us to study the thermal evolution of the Tb-moment and the unit cell parameters by using the same collinear ferromagnetic model. On figure 9, showing the temperature dependence of these parameters, we observe an interesting anisotropic magnetostriction effect at the Curie temperature: whereas the $a$ parameter decreases with decreasing temperature, the $c$ parameter increases below $\mathrm{T}_{\mathrm{C}}$ till $200 \mathrm{~K}$ and then decreases normally.

It is noteworthy that the magnetic structure of HT-TbTiGe is similar to those of closely related compounds crystallizing in the CeScSi-type structure. For examples, the compounds $\operatorname{TbTiGeC}_{\mathrm{x}}\left(\mathrm{T}_{\mathrm{C}}=310(5) \mathrm{K}\right), \operatorname{TbTiGeB}_{\mathrm{x}}\left(\mathrm{T}_{\mathrm{C}}=301(5) \mathrm{K}\right), \mathrm{TbTi}_{0.85} \mathrm{Mo}_{0.15} \mathrm{Ge}\left(\mathrm{T}_{\mathrm{C} 1}=280(3) \mathrm{K}\right.$, canted below $\left.\mathrm{T}_{\mathrm{C} 2}=75(3) \mathrm{K}\right), \mathrm{TbScGe}\left(\mathrm{T}_{\mathrm{C}}=216 \mathrm{~K}\right)$ and $\mathrm{TbZrSb}\left(\mathrm{T}_{\mathrm{C}}=65 \mathrm{~K}\right)$ also exhibit a ferromagnetic structure with the $c$-axis as easy magnetization direction [16-21]. We observe similar values for the Tb-moment around $9 \mu_{\mathrm{B}}$ except for $\mathrm{TbZrSb}$ for which $\mu_{\mathrm{Tb}}=5.6 \mu_{\mathrm{B}}$ [21]. On the contrary, for the antiferromagnetic isotype compounds $\mathrm{TbMgSn}$ and $\mathrm{TbMgPb}$, the orientation of the Tb-moments is within the basal $(a, b)$-plane [18, 22, 23]. This result is explained by the modification of the crystal electric field (CEF) interaction via the sign of the second order crystal field term $\mathrm{B}_{2}{ }^{0}=\alpha_{\mathrm{j}}<\mathrm{r}^{2}>\left(1-\sigma_{2}\right) \mathrm{K}_{2}{ }^{0} \mathrm{~A}_{2}{ }^{0}$, depending on the signs of the 
Stevens factor $\alpha_{\mathrm{j}}$ and of $\mathrm{A}_{2}{ }^{0}$ determined by the electrostatic field experienced at the Tb site due to the environment $[24,25]$. The Stevens factor depending only on the rare earth element (negative for $\mathrm{Tb}$ ), the change of the easy magnetization direction for isotype compounds results from the modification of the $\mathrm{CEF}$ on the $\mathrm{Tb}$ site due to local environment, in particular due to the nature of the transition metal. Indeed, $\mathrm{TbTi}_{0.85} \mathrm{Mo}_{0.15} \mathrm{Ge}$ presents for example, a canted ferromagnetic structure below $\mathrm{T}_{\mathrm{C} 2}=75(3) \mathrm{K}$ [19], i.e. a slight reorientation of the magnetic moment compared with HT-TbTiGe, which must reflect the influence of the substitution of Mo for Ti on the CEF.

In the low temperature form LT-TbTiGe crystallizing in the CeFeSi-type structure, the Tb-moments are also parallel to the $c$-axis with $\mu_{\mathrm{Tb}}=9.0(6) \mu_{\mathrm{B}}$ at $2 \mathrm{~K}$ but the structure is antiferromagnetic [6]: the magnetic cell is doubled along the $c$-direction and the Tb-moments are coupled in the sequence $(++--)$. Therefore, the magnetic interactions are ferromagnetic within the layers of $\left[\mathrm{Tb}_{4}\right]$ tetrahedra in both forms HT- and LT-TbTiGe, but are antiferromagnetic between these layers in LT-TbTiGe contary to those in HT-TbTiGe. This is related to very similar distances $\mathrm{d}_{\mathrm{Tb}-\mathrm{Tb}}$ within these $\mathrm{Tb}$ layers in both forms and very different distances d' ${ }_{\mathrm{Tb}-\mathrm{Tb}}$ between these layers since d' ${ }_{\mathrm{Tb}-\mathrm{Tb}}$ distances decrease drastically from 598 to $528 \mathrm{pm}$ in the sequence LT-TbTiGe $\rightarrow$ HT-TbTiGe.

\subsection{Magnetocaloric properties}

To determine the magnetocaloric properties (MCE) of HT-TbTiGe, isothermal magnetization measurements were performed around its Curie temperature $\mathrm{T}_{\mathrm{C}}$ (Figure 10). The $M$ versus $\mu_{0} H$ measurements have been carried out with increasing magnetic field from 0 to $5 \mathrm{~T}$ and for various temperatures between 330 and $100 \mathrm{~K}$ with a temperature step of $\Delta \mathrm{T}=5 \mathrm{~K}$ around $\mathrm{T}_{\mathrm{C}}$ $=300 \mathrm{~K}$. In this temperature range, no remanent magnetization persists, which should not disturb the MCE determination.

The isothermal magnetic entropy change $\Delta S_{m}$ was determined from the magnetization data by using the Maxwell relation:

$$
\Delta S_{m}(T, H)=\int_{0}^{H}\left(\frac{\partial M(T, H)}{\partial T}\right)_{H} d H
$$

The results for $\mu_{0} \Delta H=2$ and $5 \mathrm{~T}$ are reported in Figure 11. A single maximum centered between 290 and $295 \mathrm{~K}$, i.e. close to $\mathrm{T}_{\mathrm{C}}$, is observed, as expected for the magnetocaloric effect. $\Delta S_{m}$ reaches a maximum value of $-4.3 \mathrm{JK}^{-1} \mathrm{~kg}^{-1}$ for $\mu_{0} \Delta H=5 \mathrm{~T}$ and $-2.0 \mathrm{JK}^{-1} \mathrm{~kg}^{-1}$ for 
$\mu_{0} \Delta H=2 \mathrm{~T}$. These values are modest but spread over a large temperature range around $\mathrm{T}_{\mathrm{C}}$, which is typical of a second order magnetic transition. Note that a similar $\Delta S_{m}$ value was found for $\operatorname{TbTiGeB}_{\mathrm{x}}\left(-4.7 \mathrm{JK}^{-1} \mathrm{~kg}^{-1}\right.$ [18]) as expected, and a slightly smaller value for $\mathrm{TbTiGeC}_{\mathrm{x}}\left(-3.5 \mathrm{JK}^{-1} \mathrm{~kg}^{-1}[26]\right)$. Also, the maximum of the magnetic entropy change for the LT-TbTiGe was found to be equal to $0.09 \mathrm{JK}^{-1} \mathrm{~kg}^{-1} \mu_{0} \Delta H=5 \mathrm{~T}$ only [16], which arises from the antiferromagnetic behaviour of this compound.

The adiabatic temperature change $\Delta \mathrm{T}_{\mathrm{ad}}$ was determined by combining the heat capacity at zero field (Figure 6) and the magnetization data through the relationship:

$$
\Delta T_{a d}(T) \approx-\frac{T}{C_{p}(T)} \Delta S_{m}(T)
$$

(This calculation method leads to overestimated $\Delta T_{a d}$ values as reported previously by Pecharsky et al. [27]. Indeed, due to the fact that $C_{p}$ was measured at zero magnetic field only, it is impossible to get a more accurate estimation of $\left.\Delta T_{a d}\right)$. The thermal evolution of $\Delta \mathrm{T}_{\mathrm{ad}}$ for HT-TbTiGe is plotted in Figure 11 for $\mu_{0} \Delta H=5$ and $2 \mathrm{~T}$. As expected, a peak around the $\mathrm{T}_{\mathrm{C}}$ temperature in the $\Delta \mathrm{T}_{\mathrm{ad}}$ versus $\mathrm{T}$ curves is observed with a maximum of $\Delta \mathrm{T}_{\mathrm{ad}}$ of 4.2 and $2.0 \mathrm{~K}$ for $\mu_{0} \Delta H=5$ and $2 \mathrm{~T}$, respectively. This MCE amounts to around $35 \%$ of that of pure gadolinium $\left(\Delta \mathrm{T}_{\mathrm{ad}}=5.8 \mathrm{~K}\right.$ for $\mu_{0} \Delta \mathrm{H}=2 \mathrm{~T}$ [28] $)$ which is the reference for the magnetocaloric compounds.

\section{Conclusion}

The structural properties of TbTiGe are dependent on the temperature of its synthesis. After melting and quenching, the high temperature modification (HT-TbTiGe) adopts the tetragonal CeScSi-type whereas after annealing at $1073 \mathrm{~K}$, the low temperature form (LT-TbTiGe) presents the tetragonal CeFeSi-type structure. This structural transition induces different magnetic behaviours for TbTiGe: (i) HT-TbTiGe is characterized by a ferromagnetic ordering below $\mathrm{T}_{\mathrm{C}}=300(1) \mathrm{K}$ and on the contrary (ii) LT-TbTiGe is an antiferromagnet below $\mathrm{T}_{\mathrm{N}}=$ $280(1) \mathrm{K}$. The investigation of these two modifications by neutron powder diffraction reveals that the Tb-moments are in both case aligned along the $c$-axis. HT-TbTiGe shows a magnetocaloric effect around the room temperature; its magnetic entropy change $\Delta S_{m}$ is equal to $-2.0 \mathrm{JK}^{-1} \mathrm{~kg}^{-1}$ for a magnetic field change of $2 \mathrm{~T}$. This $\Delta S_{m}$-value is comparable to 
that determined previously for $\operatorname{GdScGe}\left(\Delta S_{m}=1.91 \mathrm{JK}^{-1} \mathrm{~kg}^{-1}\right)$ which adopts also the CeScSitype [29].

\section{Acknowledgment}

This work was supported by the ANR (Agence Nationale pour la Recherche) through the research project "MAGCOOL" (ANR-2010-STKE-008). 


\section{References}

[1] Morozkin A V, Seropegin Yu D, Sviridov I A, Moskalev V A 1998 J. Alloys Compd 281 228

[2] Welter R, Morozkin A V, Klosek V, Vernière A, Malaman B 2000 J. Alloys Compd 307 207

[3] Chevalier B, Hermes W, Gaudin E and Pöttgen R 2010 J.Phys.: Condens. Matter 22 146003

[4] Gaudin E, Matar S F, Pöttgen R, Eul M, and Chevalier B 2011 Inorg. Chem. 5011046

[5] Nikitin S A, Tskhadadze I A, Telegina I V, Morozkin A V, Seropegin Yu D 1998 J. Magn. Magn. Mater. 182375

[6] Vernière A, Klosek V, Welter R, Venturini G, Isnard O, Malaman B 2001 J. Magn. Magn. Mater. 234261

[7] Prokes K, Tegus O, Brück E, Gortenmulder T J, de Boer F R, Buschow K H J 2001 J. Magn. Magn. Mater. 23628

[8] Rodriguez Carvajal J 1993 Physica B 19255

[9] Sears V F 1992 Neutron News 326

[10] McCusker L B, Von Dreele R B, Cox D E, Louer D and Scardi P 1999 J. Appl. Cryst. 32 36

[11] Barlet A, Genna J C, Lethuillier P 1991 Cryogenics 31801

[12] Genna J C, Biston S, Cotillard J C, Lethuillier P, Fourin C, Hostachy P, Rocca-Valero R and Roullier J 1991 Rev. Sci. Instr. 621824

[13] Sychev N I, Safonov V N, Belokurov E V, Kalishevich G I, Gel'd P V 1983 Izvestiya Vysshikh Uchebnykh Zavedenii, Fizika 2677

[14] Morozkin A V, Viting L M, Sviridov I A, Tskhadadze I A 2000 J. Alloys Compd 297168

[15] Kolomiets A V, Mudryk Ya, Stadnyk Yu, Sechovsky V 2002 J. Alloys Compd 33334

[16] Nirmala R and Malik S K 2007 European Phys. Let. 8027001

[17] Klosek V 2002 Thesis, Université Henri Poincaré-Nancy I

[18] Lemoine P 2011 Thesis, Université Henri Poincaré-Nancy I

[19] Morozkin A V, Kurbakov A I, Klosek V, Welter R 2001 J. Alloys Compd 315100

[20] Manfrinetti P, Morozkin A V, Isnard O, Henry P, Palenzona A 2008 J. Alloys Compd 45086

[21] Morozkin A V, Halich K, Welter R, Ouladdiaf B 2005 J. Alloys Compd 39334 
[22] Ritter C, Provino A, Manfrinetti P, Gschneidner Jr. K A 2011 J. Alloys Compd 5099724

[23] Lemoine P, Vernière A, Venturini G, Capelli S, Malaman B 2012 J. Magn. Magn. Mater. 324961

[24] Greedan J E and Rao V U S 1973 J. Solid State Chem 6387

[25] Stevens K W H 1952 Proc. Roy. Soc. A 165372

[26] Mazet T, Recour Q, Vernière A, Malaman B 2007 Proceedings of the $2^{\text {nd }}$ International Conference of the IIR on Magnetic Refrigeration at Room Temperature, Thermag II

[27] Pecharsky V K, Gschneidner Jr K A 1999 J. Appl. Phys. 86565

[28] Dan'kov S Yu, Tishin A M, Pecharsky V K and Gschneidner Jr K A 1998 Phys. Rev. B. 573478

[29] Couillaud S, Gaudin E, Franco V, Conde A, Pöttgen R, Heying B, Rodewald U Ch, Chevalier B, 2011 Intermetallics 191573 
Table 1. Lattice, positional and isotropic displacement parameters at room temperature for HT-TbTiGe (CeScSi-type structure with space group $14 / \mathrm{mmm}$ ) as deduced from Rietveld refinement of X-ray powder diffraction (first line) and neutron powder diffraction (second line)

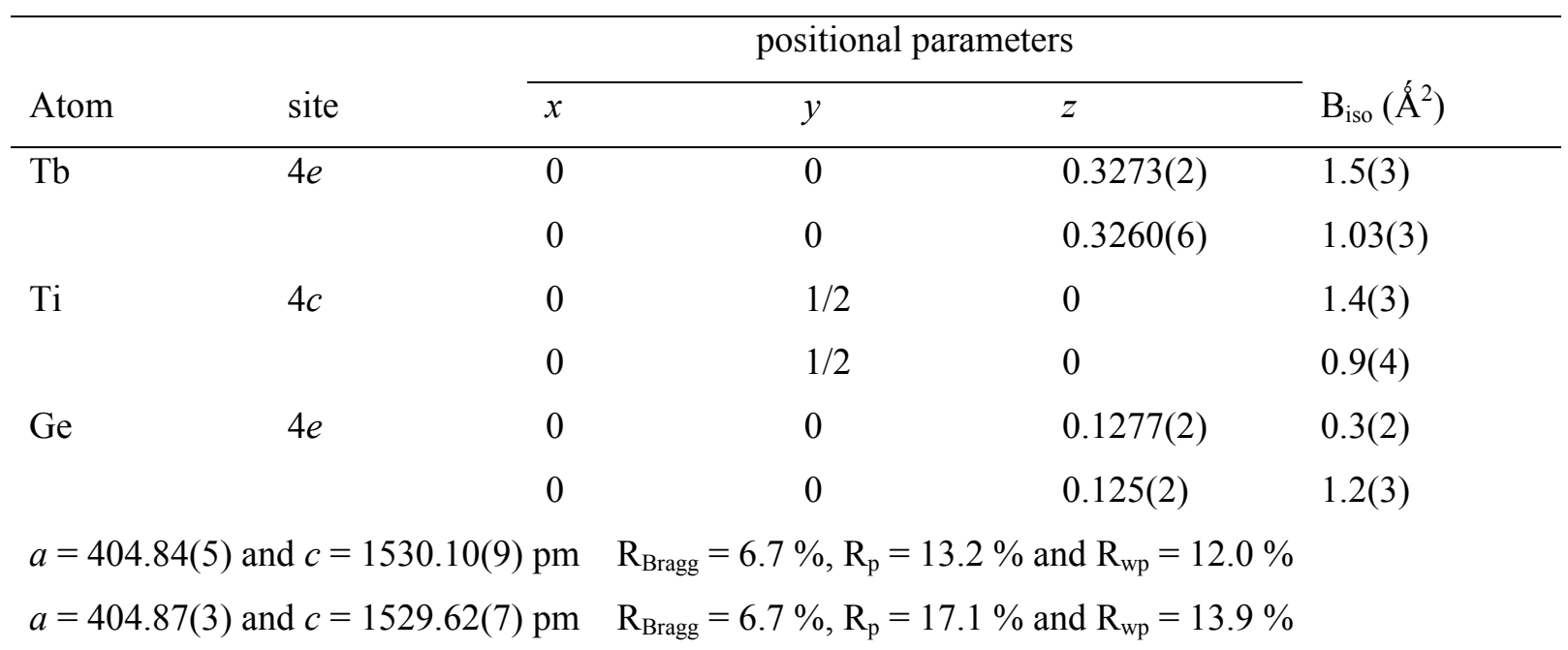




\section{Figure captions}

Figure 1. Low angle part of the X-ray powder patterns of the TbTiGe sample after melting (as-cast) followed by annealing at $1073 \mathrm{~K}$ (annealed) characteristic of the HT-form and the LT-form respectively. The patterns have been shifted vertically for clarity.

Figure 2. Rietveld profile refinement of the X-ray powder pattern recorded at room temperature for the HT-TbTiGe ternary germanide $\left(\lambda=1.54059 \AA\right.$ Á). The $\mathrm{Ti}_{5} \mathrm{Ge}_{3}$ phase was included in the refinement. The observed data, the calculated profile and the difference between observation and calculation are given respectively in red circles, black and blue (bottom) solid lines. The green ticks correspond to $2 \theta$-Bragg peak positions of HT-TbTiGe (1) and $\mathrm{Ti}_{5} \mathrm{Ge}_{3}(2)$.

Figure 3. Temperature dependence of the magnetization $M$ of HT-TbTiGe measured under an applied magnetic field $H=0.05 \mathrm{~T}$ determined in the zero field cooling (ZFC) and in the field cooling (FC) process. The inset presents the thermal dependence of $M$ recorded for the LTTbTiGe phase.

Figure 4. Temperature dependence of the reciprocal magnetic susceptibility $\chi_{\mathrm{m}}{ }^{-1}$ of HTTbTiGe measured in an applied magnetic field of $0.1 \mathrm{~T}$. The dashed line presents the CurieWeiss law.

Figure 5. Field dependence at $10 \mathrm{~K}$ of the magnetization $\mathrm{M}$ of the ternary germanide HTTbTiGe.

Figure 6. Temperature dependence of the specific heat $C_{p}$ of the ternary germanide HTTbTiGe.

Figure 7. Comparison of the magnetic structure obtained at $2 \mathrm{~K}$ for the two inequivalent crystal structures: LT-TbTiGe (a) and HT-TbTiGe (b). 
Figure 8. Rietveld refinement of the neutron diffraction pattern at $2 \mathrm{~K}$ of HT-TbTiGe $(\lambda=$ $2.52 \AA$ Á). The observed data, the calculated profile and the difference between observation and calculation are given respectively in red circles, black and blue (bottom) solid lines. The green ticks correspond to $2 \theta$-Bragg peak positions of HT-TbTiGe (nuclear), HT-TbTiGe (magnetic) and $\mathrm{Ti}_{5} \mathrm{Ge}_{3}$ from top to bottom respectively.

Figure 9. Temperature dependence of the Tb-moment (upper part) and of the unit cell parameters (lower part) as deduced from the analysis of the neutron diffraction data.

Figure 10. Field dependence at various temperature $(100 \mathrm{~K} \leq \mathrm{T} \leq 330 \mathrm{~K})$ of the magnetization $\mathrm{M}$ of the ternary germanide HT-TbTiGe.

Figure 11. Temperature dependence for $\mu_{0} \Delta H=2$ and $5 \mathrm{~T}$ of the isothermal magnetic entropy change $\Delta S_{m}$ (upper part) and of the adiabatic temperature change $\Delta \mathrm{T}_{\mathrm{ad}}$ (lower part) for HTTbTiGe. 


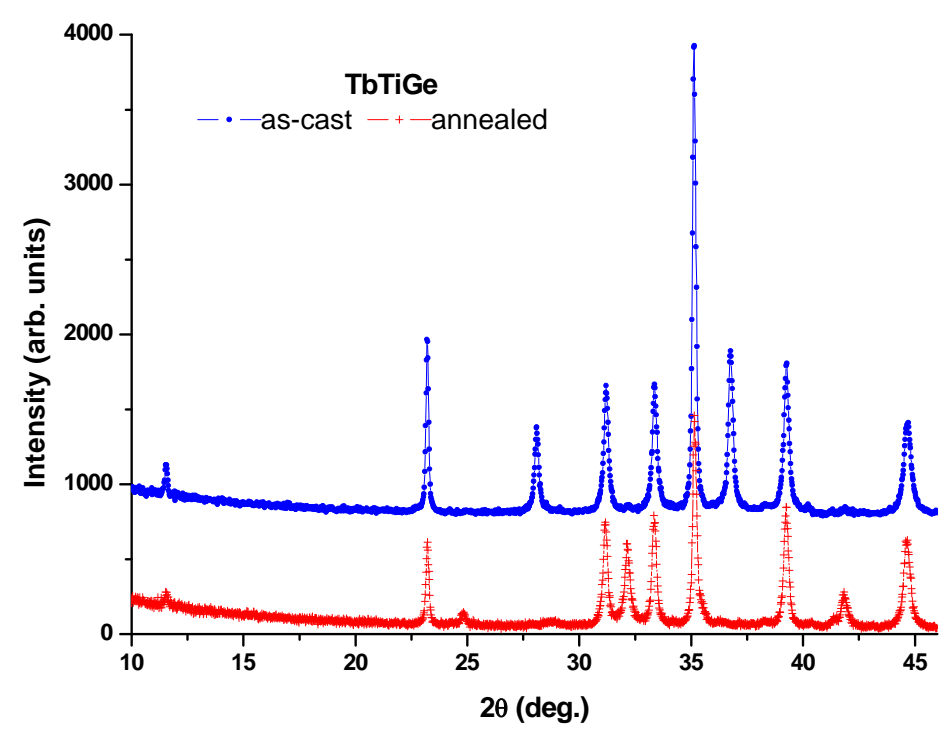

Figure 1. Low angle part of the X-ray powder patterns of the TbTiGe sample after melting (as-cast) followed by annealing at $1073 \mathrm{~K}$ (annealed) characteristic of the HT-form and the LT-form respectively. The patterns have been shifted vertically for clarity. 


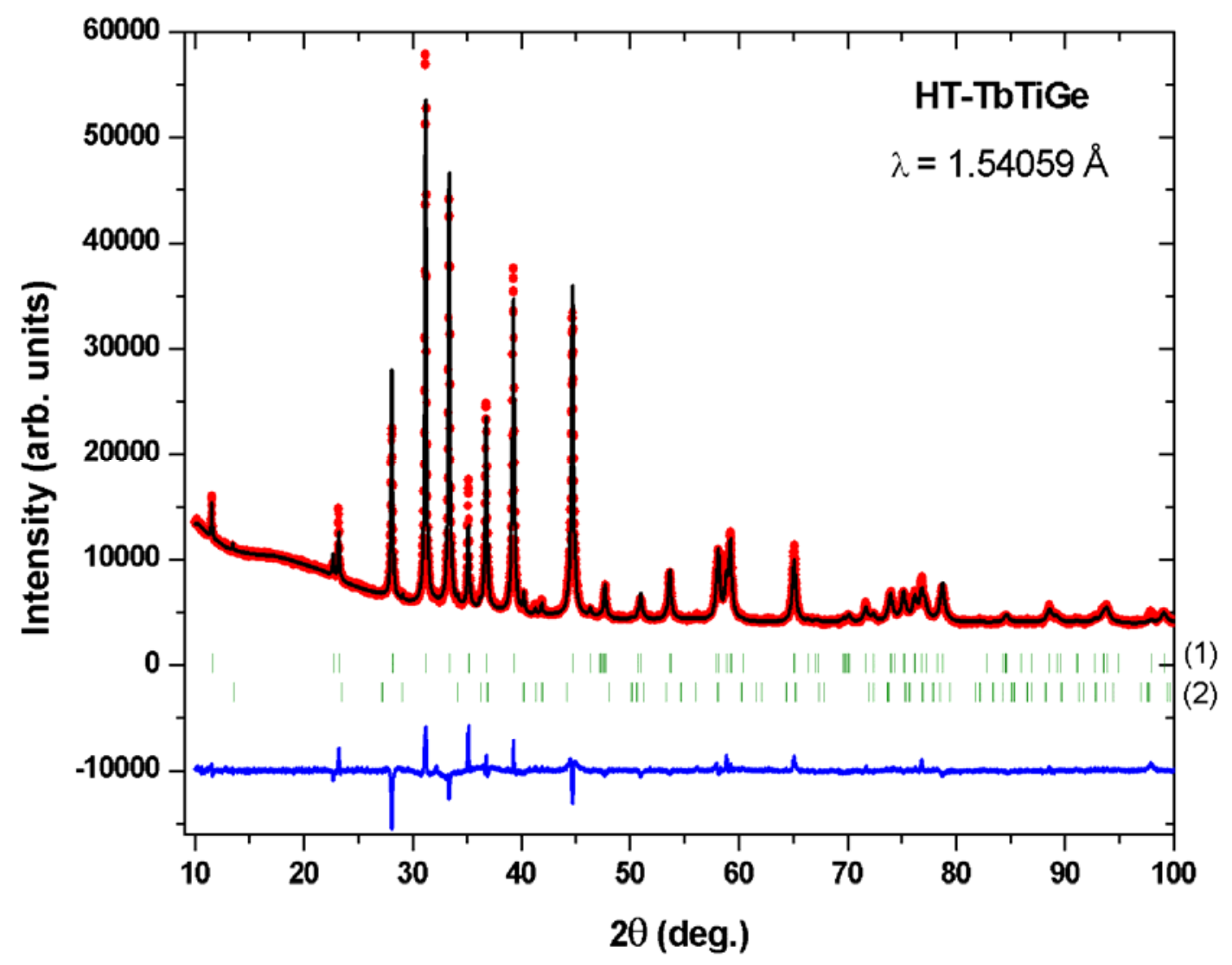

Figure 2. Rietveld profile refinement of the X-ray powder pattern recorded at room temperature for the HT-TbTiGe ternary germanide $(\lambda=1.54059 \AA)$ ). The $\mathrm{Ti}_{5} \mathrm{Ge}_{3}$ phase was included in the refinement. The observed data, the calculated profile and the difference between observation and calculation are given respectively in red circles, black and blue (bottom) solid lines. The green ticks correspond to $2 \theta$-Bragg peak positions of HT-TbTiGe (1) and $\mathrm{Ti}_{5} \mathrm{Ge}_{3}(2)$. 


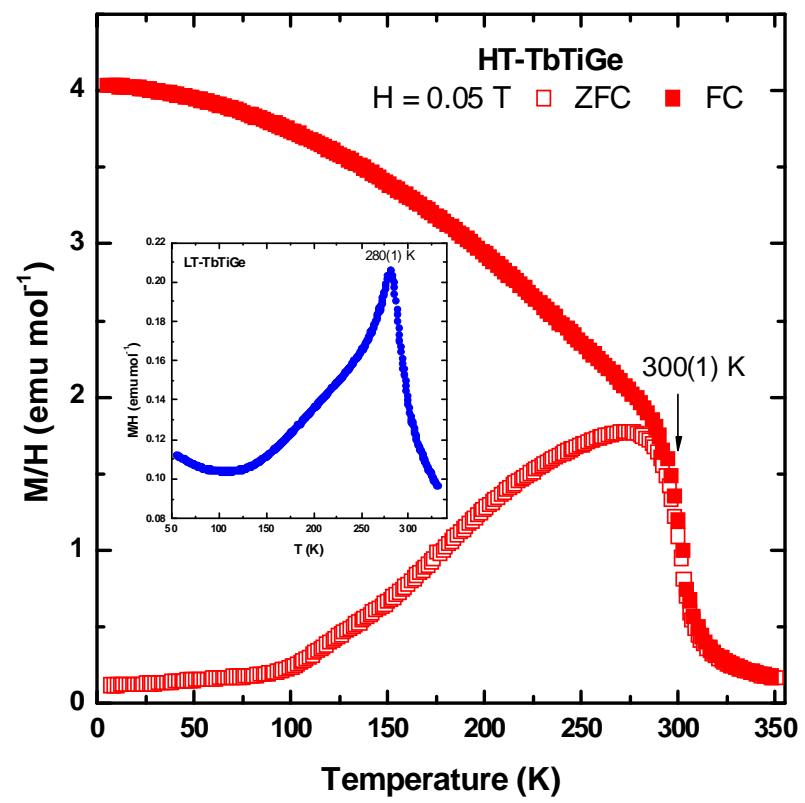

Figure 3. Temperature dependence of the magnetization $M$ of HT-TbTiGe measured under an applied magnetic field $H=0.05 \mathrm{~T}$ determined in the zero field cooling (ZFC) and in the field cooling (FC) process. The inset presents the thermal dependence of $M$ recorded for the LTTbTiGe phase. 


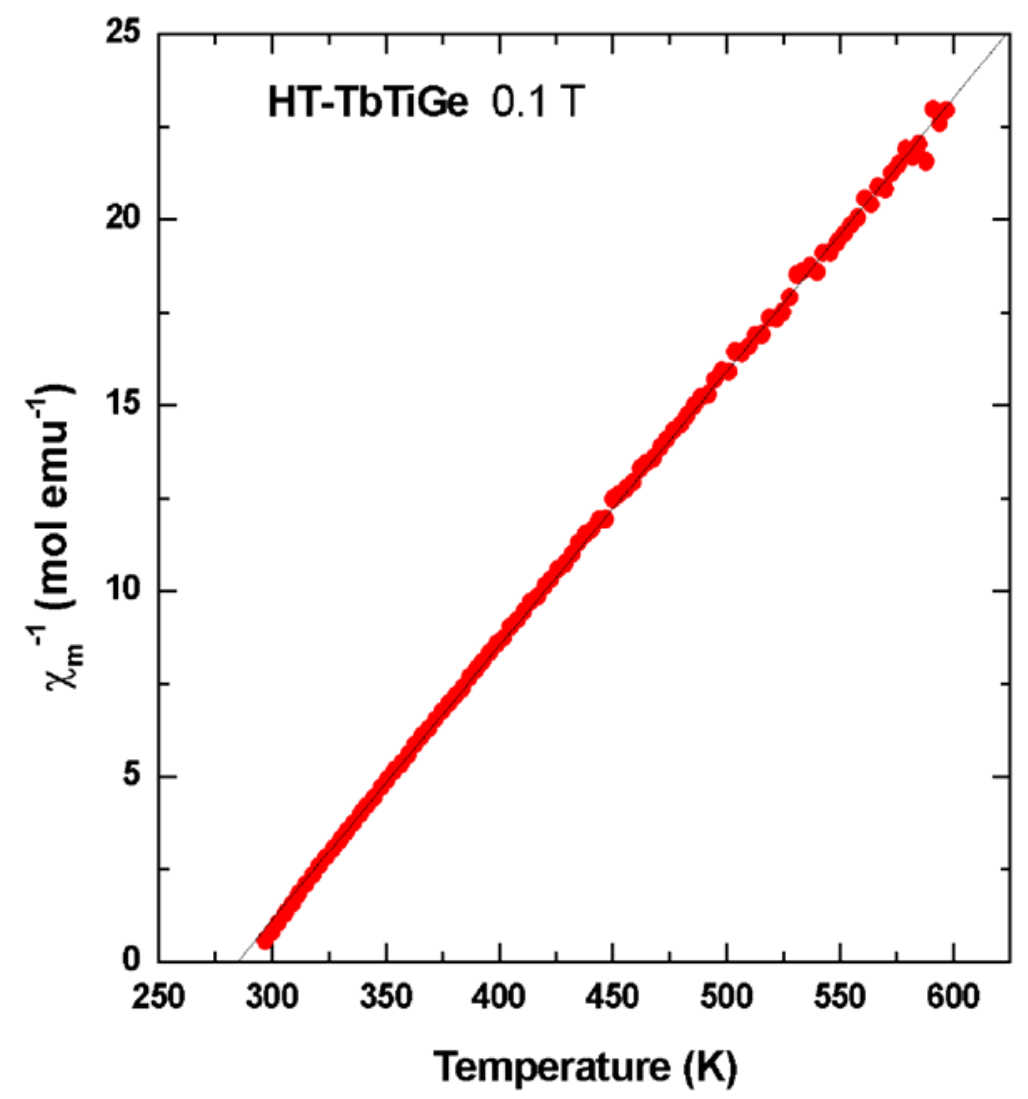

Figure 4. Temperature dependence of the reciprocal magnetic susceptibility $\chi_{\mathrm{m}}{ }^{-1}$ of HTTbTiGe measured in an applied magnetic field of $0.1 \mathrm{~T}$. The dashed line presents the CurieWeiss law. 


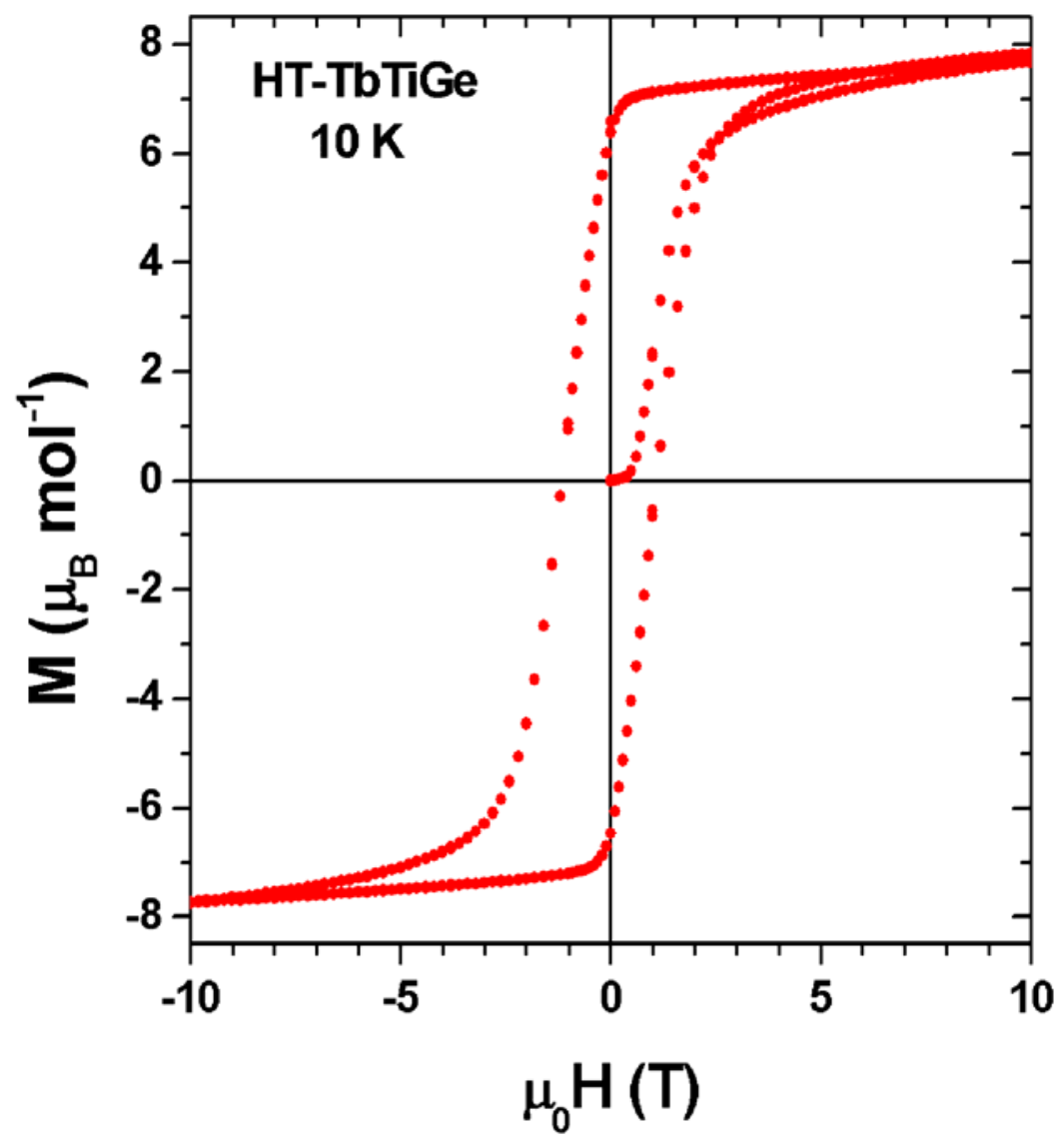

Figure 5. Field dependence at $10 \mathrm{~K}$ of the magnetization $M$ of the ternary germanide HTTbTiGe. 


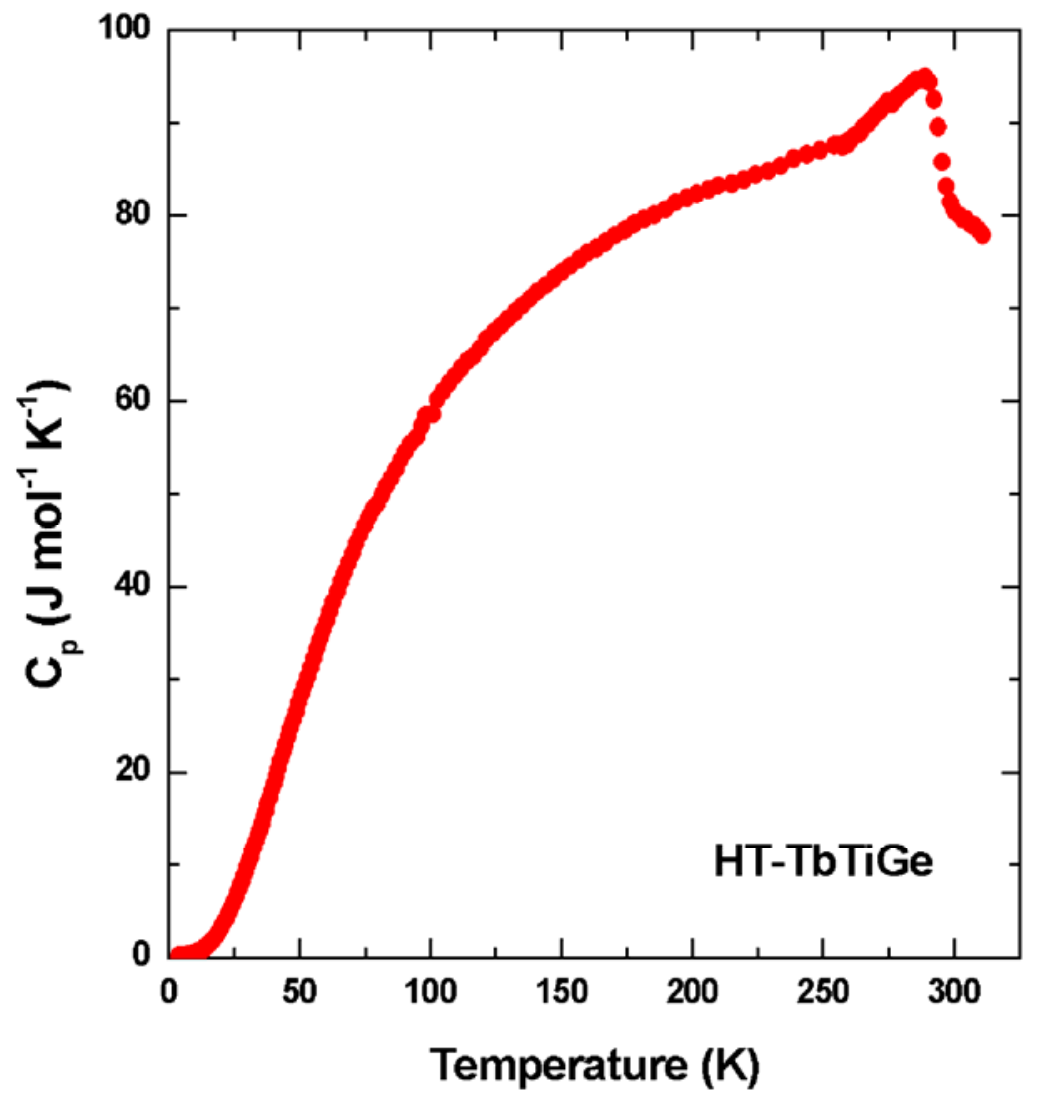

Figure 6. Temperature dependence of the specific heat $C_{p}$ of the ternary germanide HTTbTiGe. 
(a)

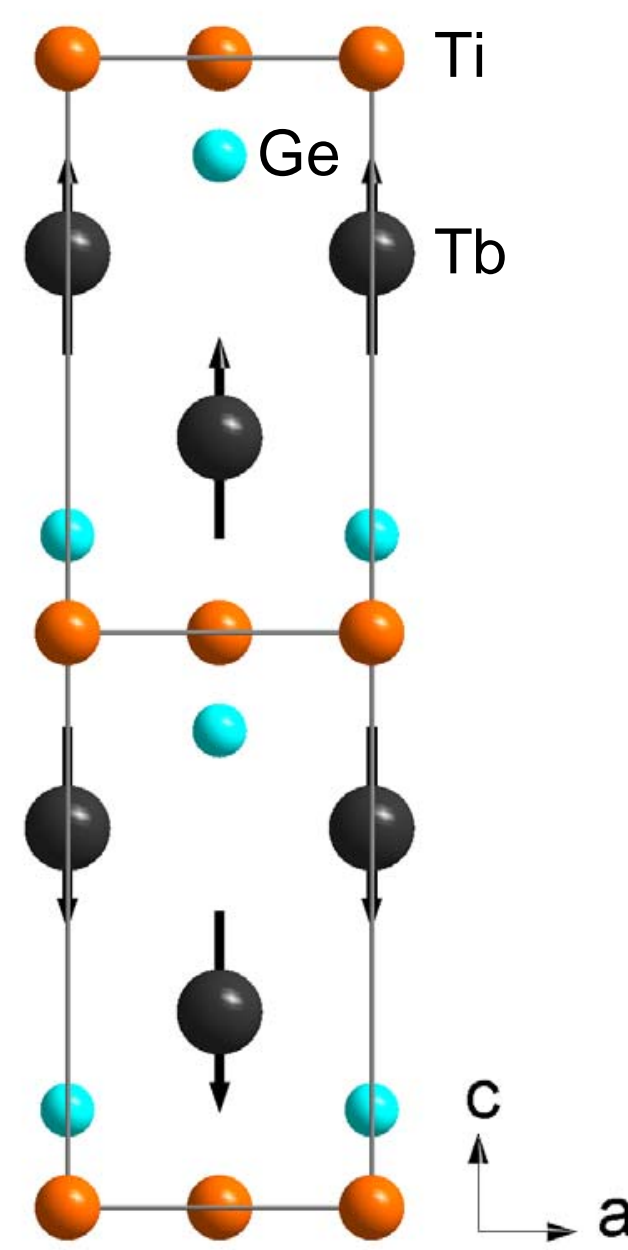

(b)

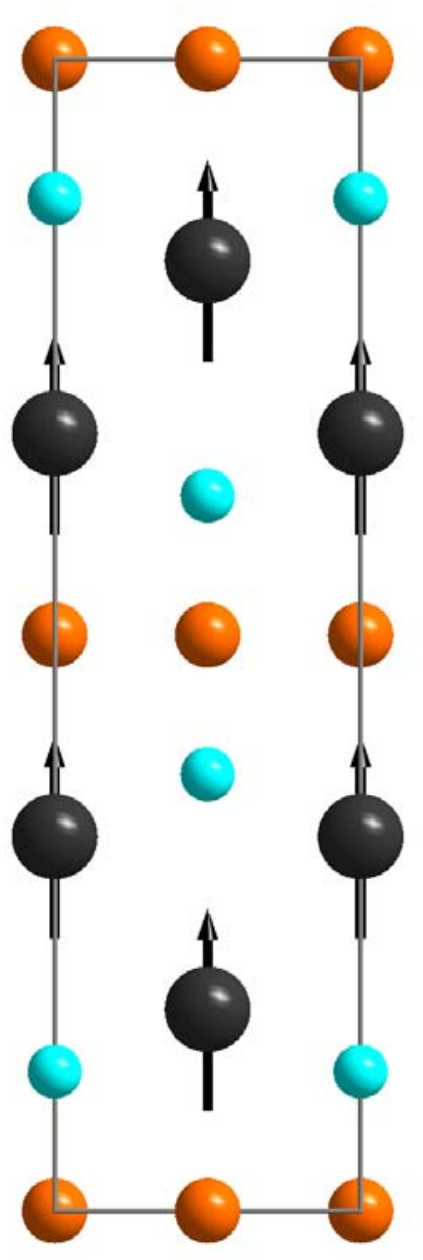

Figure 7. Comparison of the magnetic structure obtained at $2 \mathrm{~K}$ for the two inequivalent crystal structures: LT-TbTiGe (a) and HT-TbTiGe (b). 


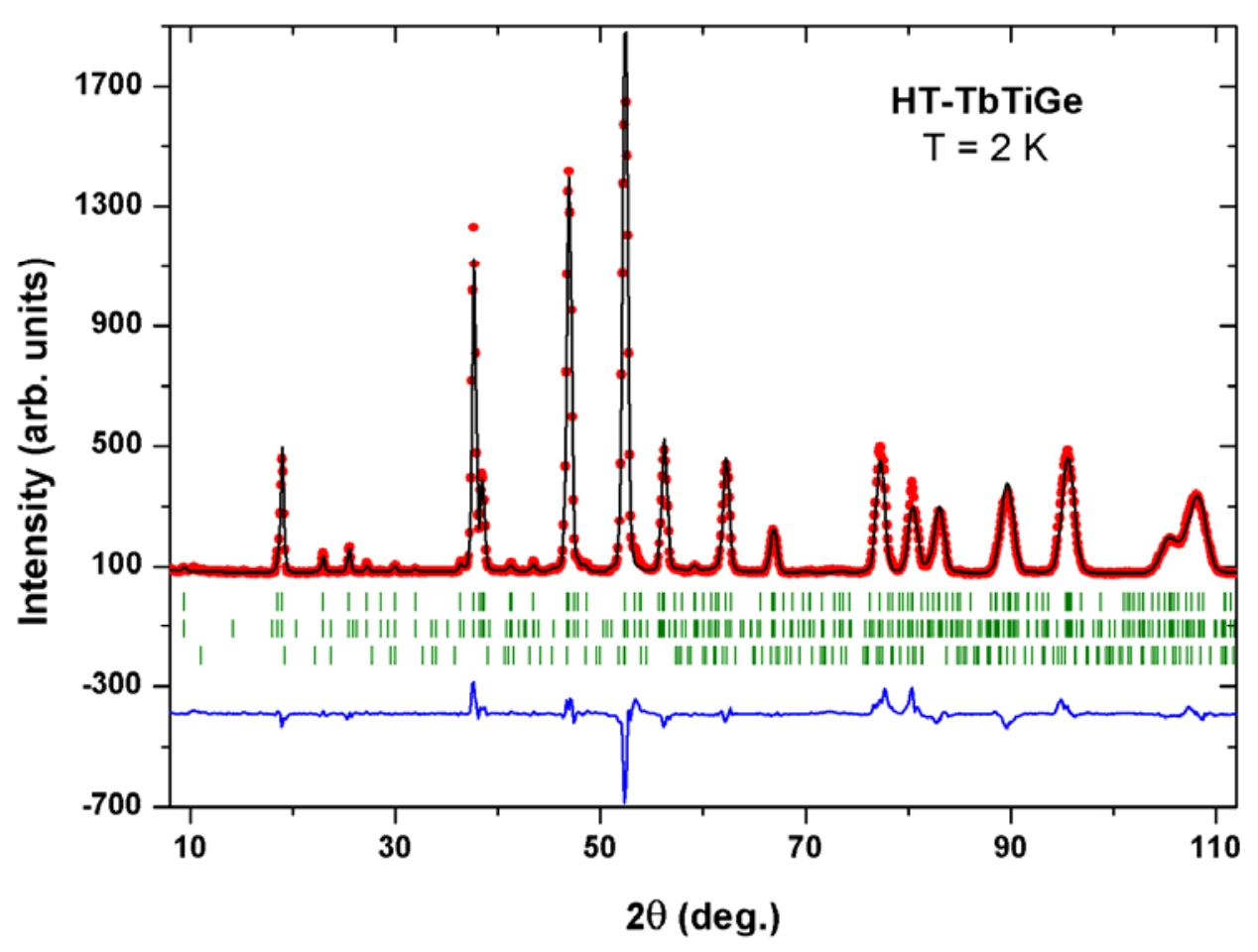

Figure 8. Rietveld refinement of the neutron diffraction pattern at $2 \mathrm{~K}$ of $\mathrm{HT}-\mathrm{TbTiGe}(\lambda=$ $2.52 \AA$ ). The observed data, the calculated profile and the difference between observation and calculation are given respectively in red circles, black and blue (bottom) solid lines. The green ticks correspond to $2 \theta$-Bragg peak positions of HT-TbTiGe (nuclear), HT-TbTiGe (magnetic) and $\mathrm{Ti}_{5} \mathrm{Ge}_{3}$ from top to bottom respectively. 

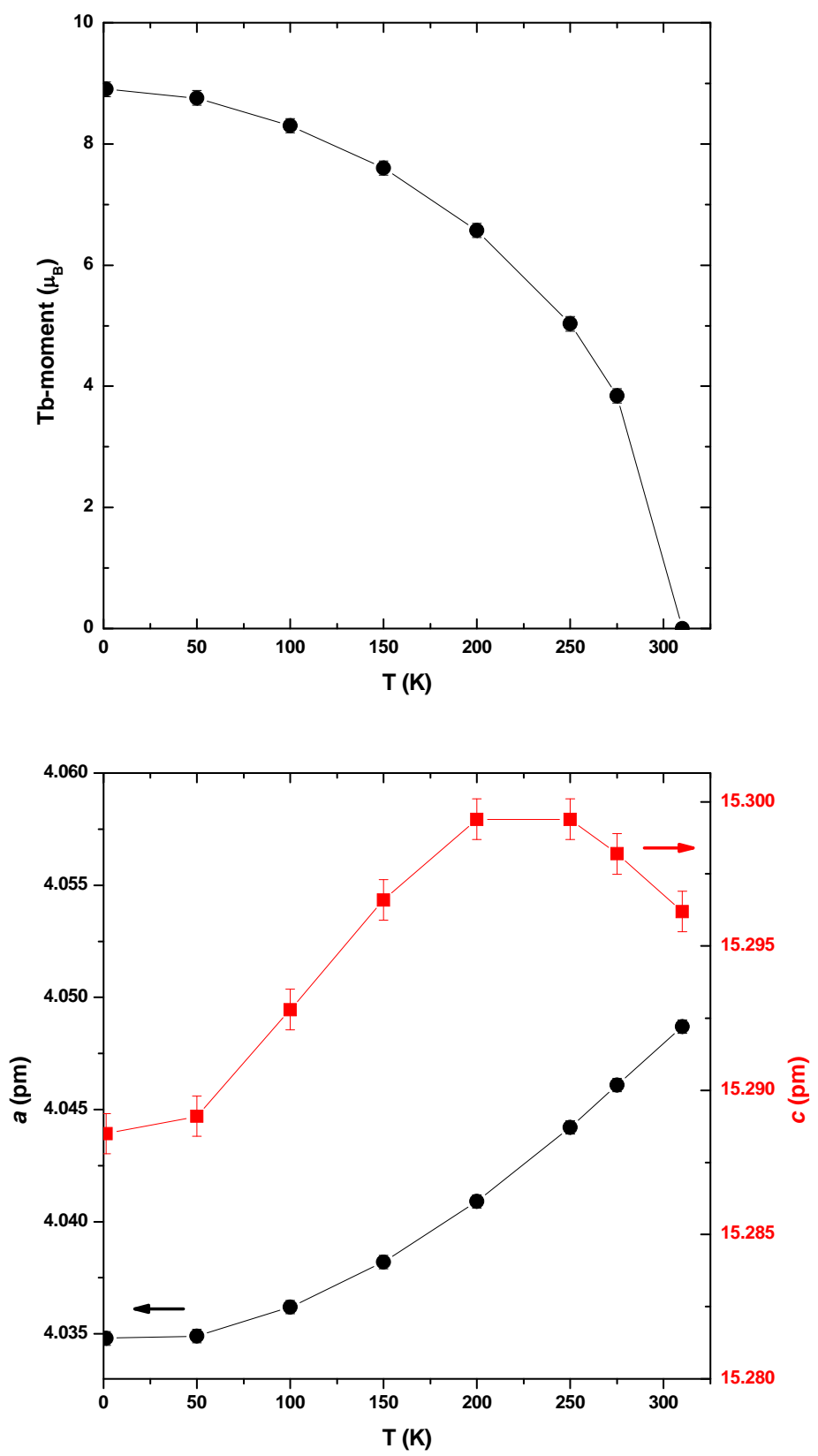

Figure 9. Temperature dependence of the Tb-moment (upper part) and of the unit cell parameters (lower part) as deduced from the analysis of the neutron diffraction data. 


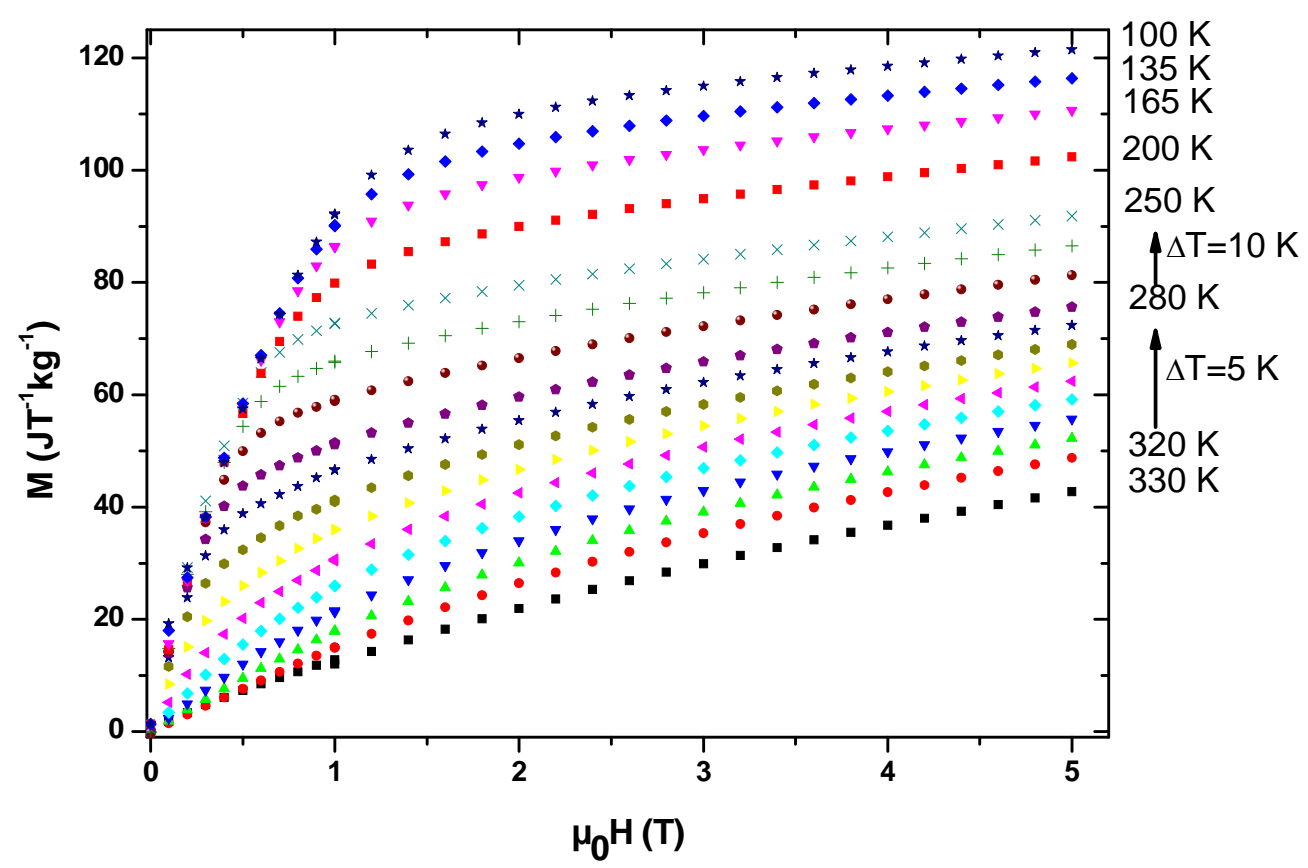

Figure 10. Field dependence at various temperature $(100 \mathrm{~K} \leq \mathrm{T} \leq 330 \mathrm{~K})$ of the magnetization $\mathrm{M}$ of the ternary germanide HT-TbTiGe. 

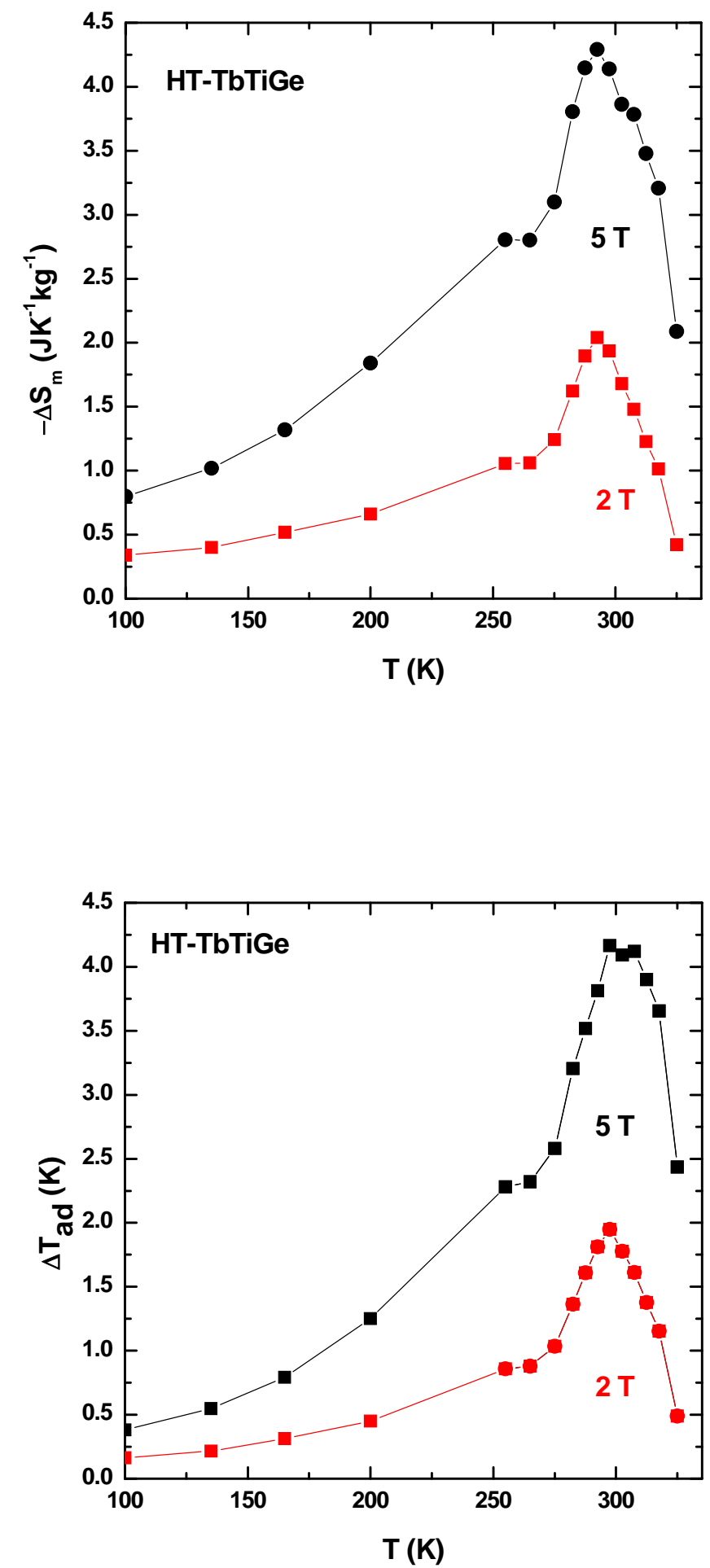

Figure 11. Temperature dependence for $\mu_{0} \Delta H=2$ and $5 \mathrm{~T}$ of the isothermal magnetic entropy change $\Delta S_{m}$ (upper part) and of the adiabatic temperature change $\Delta \mathrm{T}_{\mathrm{ad}}$ (lower part) for HTTbTiGe. 